\title{
Real-Space Visualization of Energy Loss and Carrier Diffusion in Semiconductor Nanowire Array using Four-Dimensional Electron Microscopy
}

Riya Bose, ${ }^{\S, 1}$ Jingya Sun, ${ }^{\S, 1}$ Jafar I. Khan, ${ }^{1}$ Basamat S. Shaheen, ${ }^{1}$ Aniruddha Adhikari, ${ }^{1}$ Tien

Khee Ng, ${ }^{2}$ Victor M. Burlakov, ${ }^{3}$ Manas R. Parida, ${ }^{1}$ Davide Priante, ${ }^{2}$ Alain Goriely, ${ }^{3}$ Boon S. Ooi, ${ }^{2}$ Osman M. Bakr, ${ }^{1}$ Omar F. Mohammed ${ }^{* 1}$

Dr. R. Bose, Dr. J. Sun, Dr. J. I. Khan, Dr. A. Adhikari, B. S. Shaheen, Prof. O. M. Bakr, Prof. O. F. Mohammed, Solar and Photovoltaics Engineering Research Center, Division of Physical Sciences and Engineering, King Abdullah University of Science and Technology, Thuwal 23955-6900, Kingdom of Saudi Arabia, D. Priante, Dr. T. K. Ng, Prof. B. S. Ooi, Photonics Laboratory, Computer, Electrical, and Mathematical Sciences and Engineering KAUST, Thuwal, 23955-6900, Kingdom of Saudi Arabia

Dr. V. M. Burlakov and Prof. A. Goriely, Mathematical Institute, University of Oxford, Woodstock Road, Oxford OX2 6GG, UK,

KEYWORDS. Ultrafast electron microscopy, real-space imaging, carrier dynamics, energy loss, carrier diffusion, surface dynamics 
By combining the nanometer spatial resolution of the electron microscope (EM) with ultrafast electron pulses, the invention of so-called four-dimensional ultrafast electron microscopy (4D UEM) has indeed unraveled a new dimension in imaging techniques that enables the space-time visualization of non-equilibrium electronic and structural dynamics. ${ }^{[1-3]}$ In a UEM experiment, single electron pulses, which are generated by fs laser pulses, are utilized and further synchronized with optical-excitation pulses, providing a new, powerful methodology to image ultrafast dynamical processes in real space and time. In this regime, the replacement of a continuous electron beam, as used in conventional EM, by photoelectron packets, for which the probe pulse contains at most a few electrons, can significantly eliminate Coulomb repulsion between electrons and allows the temporal resolution of UEM to be determined only by the optical pulse duration, while maintaining the same spatial resolution of EM. ${ }^{[1-4]}$ Amongst the different techniques of microscopy that have been integrated into UEM, ${ }^{[5-10]}$ S-UEM is particularly befitting of selectively probing surface dynamics in real space and time. ${ }^{[6,11-13]}$ More specifically, in S-UEM, time-resolved secondary electron (SE) images are generated from the specimen surface following in situ fs optical excitation (see Figure 1) and probing with a pulsed primary electron beam. This allows for the study of physical and chemical dynamics on the surface and at the interface of materials. ${ }^{[6,11-12,14]}$ Recently, ultrafast transient absorption microscopy techniques have also been developed to examine charge carrier dynamics of nanostructures with high spatial and temporal resolution; ${ }^{[15-16]}$ however, these techniques are limited by the laser's relatively large penetration depth, and therefore they record mainly bulk information.

The ultrafast dynamics of charge carriers on materials surfaces plays a pivotal role in controlling the applicability of nanoscale materials, especially for one-dimensional 
nanostructures, e.g., nanowires/nanorods, which have been extensively used for electronic, optoelectronic and photoelectrochemical applications due to their interesting and unique properties. ${ }^{[17-28]}$ For instance, an understanding of non-radiative energy losses at the surface, via carrier-carrier and carrier-phonon interactions, following pulsed-laser excitation is crucial for wide bandgap semiconductors, e.g., group III nitride materials and alloys. ${ }^{[29-33]}$ Of particular interest is the InGaN system that has found wide-spread use as the active media in solid state light emitting diodes, laser diodes and photovoltaic devices because of its tunable bandgap from a value of 0.7 to $3.4 \mathrm{eV}$ and other characteristics such as a direct band gap, high absorption coefficient and irradiance resistance. ${ }^{[34-38]}$ However, the internal quantum efficiency (IQE) of these devices is limited by an energy loss mechanism, which leads to a decrease in the IQE at high driving currents. This phenomenon is commonly known as an "efficiency droop". ${ }^{[39-41]}$ The precise nature of this energy loss mechanism has been the subject of intense debate, and a variety of pathways have been proposed. Intra-and inter-band Auger recombination events, which involve the scattering of an electron within the lowest and to the second-lowest conduction bands, respectively, along with alloy scattering, i.e., the scattering of carriers due to the disorder component of the crystal potential, have been the most reported explanations. ${ }^{[42-46]}$ The scattering processes also limit the mobility of charge carriers, which in turn diminishes the efficiency of optoelectronic devices. ${ }^{[46-48]}$ It should also be noted that under low injection current density, the carrier diminishing mechanism in the InGaN nanowires is dominated by the surface-states related Shockley-Read-Hall recombination, a monomolecular recombination phenomenon. It was reported that a reduction in surface-states resulted in a higher peak-IQE and overall higher IQE, or the related quantity, the external quantum efficiency (EQE), even when the Auger recombination process started dominating at a higher injection current density. ${ }^{[49]}$ Therefore, to 


\section{WILEY-VCH}

unlock the full potential of InGaN for efficient device-based applications, a precise knowledge of the surface recombination dynamics is of utmost importance. The limited penetration depth of the electron beam in SUEM, as has been discussed, warrants information about charge carrier recombination and diffusion process dominated mostly by surface states, and will indirectly shed light on the improvement in IQE at low injection current density regime of operation.

Herein, we image the charge carrier dynamics of a densely packed array of InGaN nanowires (NWs) oriented vertically on the substrate with the length and the diameter being in the range of 460-510 $\mathrm{nm}$ and 20-40 $\mathrm{nm}$ respectively, upon fs optical excitation using S-UEM. Unlike the cases of CdSe single crystals and $\mathrm{Si},{ }^{[11]}$ where bright contrast has been observed at positive time delays, the measured contrast in time-resolved SE images at both negative and positive time delays is dark. From the time scale obtained, we are able to deduce the deactivation channels and the mechanism of SE energy loss. Interestingly, imaging the signal spread over a time window of 6.0 ns provides clear experimental evidence for the diffusion of charge carriers across the boundaries of the nanowire array, which is indeed a critical parameter to control the efficiency of InGaN light emitting devices and laser diodes. ${ }^{[00-52]}$

The schematic diagram for 4D-SUEM is provided in Figure 1, and detailed information on the principle of operation is published elsewhere. ${ }^{[11,13]}$ Briefly, the output of a fs Clark-MXR fiber laser system is integrated with a modified Quanta FEI-650 SEM. The laser delivers IR pulses centered at $1030 \mathrm{~nm}$ with a pulse width of $\sim 270 \mathrm{fs}$ and the repetition rate ranges from $200 \mathrm{KHz}$ to $25.4 \mathrm{MHz}$. The fundamental output is split by a 1:1 beam splitter, and thus it simultaneously pumps two second/third harmonic generators (HG, Clark-MXR) to produce 515 or $343 \mathrm{~nm}$ pulses. The $343 \mathrm{~nm}$ output is directed and tightly focused onto the emitter tip, which photogenerates electron pulses that are then accelerated using a $30 \mathrm{keV}$ voltage. The $515 \mathrm{~nm}$ output 
enters the microscope through a viewport that is at a 50-degree angle relative to the surface normal and is focused on the specimen for dynamics initiation. The scanning process of the electron beam takes place across the surface of the sample, both the laser excited and unexcited regions in raster pattern, and the SEs emitted from the sample are detected by a positively biased Everhart-Thornley detector. The SEs images were obtained as integration of 64 frames with dwell time of $300 \mathrm{~ns}$ at each pixel to improve the signal-to-noise ratio. Finally, all experiments are conducted at a repetition rate of $8 \mathrm{MHz}$ to ensure a full recovery of the specimen before the arrival of the next pulse.

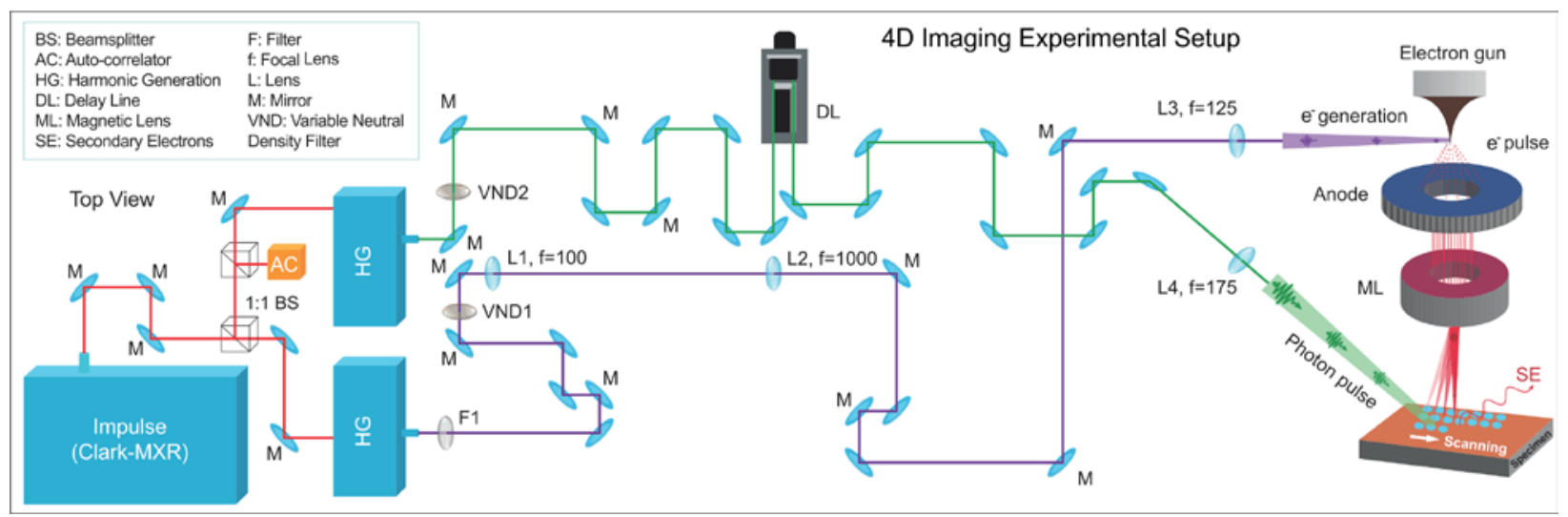

Figure 1. A conceptual schematic of the S-UEM. Pulsed electrons are scanned over the surface that is illuminated by an optical pulse. The emitted SEs are then detected to construct timeresolved SEs images at different time delays between the optical and electron pulses.

Figure 2 shows the time-resolved SE images that are obtained from InGaN NWs at different time delays in response to the $515 \mathrm{~nm}$ excitation pulse. As can clearly be seen, a dark contrast is observed in the laser-illuminated region when the electron pulse arrives before the clocking pulse near time zero. At positive time delays, the measured contrast is also dark, which suggests that the SEs generated in both regimes lose energy during their migration to the surface in order to escape (details described below). It should be noted that the dark contrast spreads beyond the 
laser footprint region with time, which originates from carrier diffusion from the laser illuminated part. Dense packing of the NW array can enable the carrier diffusion across the boundaries of the NWs, and for ease of understanding, the sample can be compared to a thin film with columnar growth.

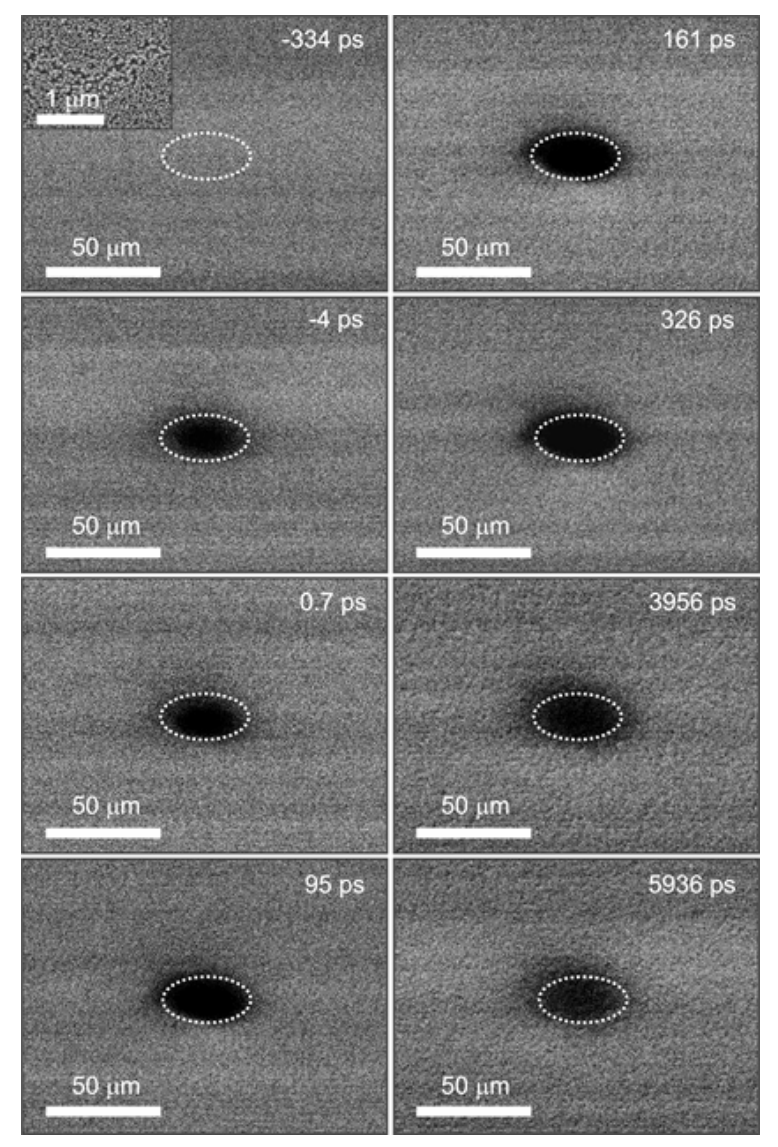

Figure 2. Time-resolved SE images of InGaN NWs at the indicated time delays. The dashed ellipses indicate the footprint of the clocking optical beam on the specimen $(\sim 40 \mu \mathrm{m})$. No observable change in the contrast at very negative time signifies the recovery of the system to an equilibrated state after each pump-probe event. An SEM image of the nanowires is shown in the inset (top panel) and cross section SEM image has been provided in Figure S2. 
To investigate the carrier dynamics, the SE intensities from the center and outside the periphery of the laser excitation footprint are plotted as functions of the time delay between optical and electron pulses (Figure 3, top panel). The pulse energy of the pump beam is $0.06 \mathrm{~nJ}$ at the sample; it is worth mentioning that we also performed the same experiment under different pulse energies $(0.012,0.036$, and $0.073 \mathrm{~nJ})$, and the kinetics at the laser footprint remain essentially the same, indicating the independence of the carrier dynamics for this range of pulse energy. It is worth pointing out that the Si substrate will not contribute to the observed contrast change due to the very low excitation pulse energies used. In addition, the long length of InGaN NWs does not allow any SEs to come out from Si substrate. For the signal collected from the center, it is observed that the intensity reaches its maximum darkness within the temporal resolution of the instrument. As time passes, in addition to the spreading of this dark contrast, the signal fades away, indicating carrier relaxation. It should be noted that the carrier relaxation is much slower than the maximum time window of the SUEM; therefore, an accurate decay time cannot be extracted from fitting the data on this time scale. On the other hand, the SE kinetics obtained from outside the periphery of the signal (ca. a few micro-meters) shows a time constant of $1.2 \pm 0.2 \mathrm{~ns}$ to reach its maximum intensity. To confirm the origin of this slow dynamic feature, we use the ambipolar diffusion coefficient for electrons in InGaN nanowires $\left(26 \mathrm{~cm}^{2} / \mathrm{sec}\right)^{[11,53]}$ to calculate the carrier diffusion/spreading away from the laser footprint region. Based on this information, we found that carriers can diffuse approximately $3.95 \mu \mathrm{m}$ within the 6 ns time window, which is in good agreement with our measurements. This observation provides clear evidence for the accessibility of probing carrier spreading on material surfaces in space and time using S-UEM. 
The observed dark contrast in the S-UEM images at negative delays with a $20 \pm 4$ ps time constant (Figure 3, Top panel; inset) can be explained as follows. The electron pulse arrives at the sample prior to the fs optical pulse (Figure 3, middle panel). ${ }^{[6,11-12]}$ The non-equilibrium change in the sample by electron impact is altered again by the following optical pulse. This dark contrast can be attributed either to the diffusion of plasmon-excited carriers that are generated from deeper areas of the sample by electron impact, which can be perturbed by electron- hole pairs generated by the clocking photon pulse, or to different scattering processes. ${ }^{[54]}$ Considering the ambipolar diffusion coefficient of $26 \mathrm{~cm}^{2} / \mathrm{sec}$ in InGaN NWs, and if we assume that $\sim 250 \mathrm{~nm}$ constitutes the region of the distribution that can eventually contribute to the SE signal, we obtain (using $\mathrm{L}^{2} / \mathrm{D}$; where $\mathrm{L}=$ the distance charge carrier diffuses and $\mathrm{D}=$ Diffusion coefficient) a time constant of about $\sim 24$ ps for the plasmon excited carriers to reach the surface. This seems to be a reasonable explanation for the observed dark contrast. On the other hand, scattering seems less likely because the characteristic time constant for electron-electron and electronphonon scattering processes are in the range of a few femtoseconds and picoseconds, respectively, which does not match the observed time. To further confirm the origin of the dark contrast in the negative time, we did two different control S-UEM experiments, by varying the pulse energy of the electron beam as well as the thickness of the sample, both of which suggest that the negative signal must have originated from plasmon excited carriers that are generated from deeper areas of the sample (Experimental details provided in Supporting Information, Figure S3-S4). 


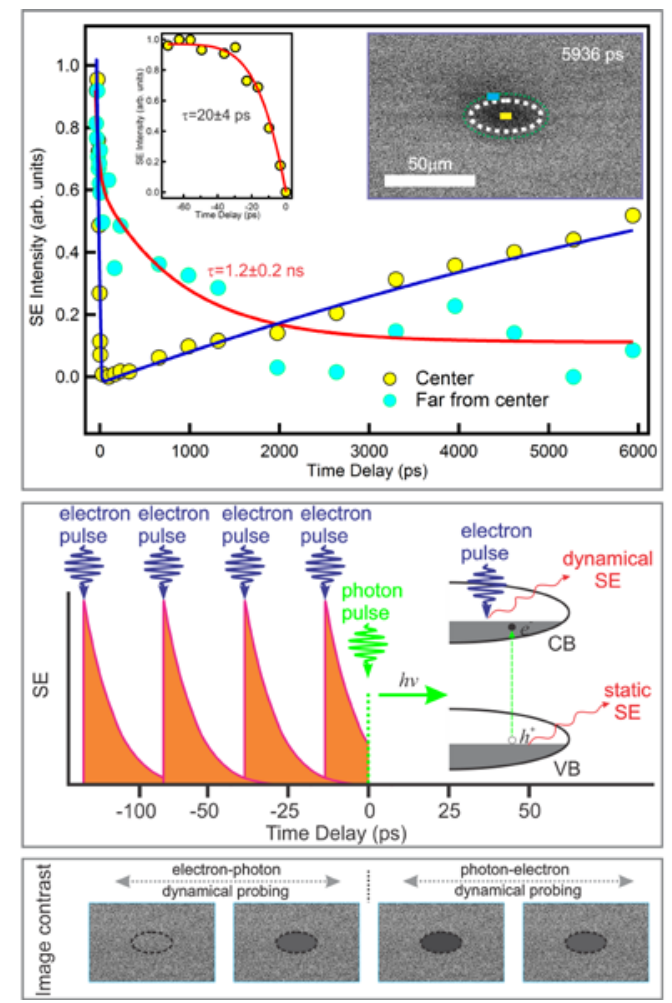

Figure 3. Top panel: The dynamics of the temporal evolution of the SE intensity at the center and outside of the laser footprint. Inset shows the SE kinetics in the negative time region. Middle panel: Schematic diagram showing the principle of imaging in negative (the electron pulse arriving at the sample prior to the optical pulse) and positive (the optical pulse is prior to the electron pulse) time scales. Bottom Panel: Shows the time regimes that are probed: electronphoton and photon-electron probing.

At positive time delays in the S-UEM experiment, the optical pulse promotes a fraction of the valence-band electrons to the conduction band (Figure 3, middle panel). Compared with electrons in an unexcited specimen, the promoted electrons have a high probability of emitting SEs to the vacuum level when they are scattered by the energetic primary electron beam, which results in an enhanced probability of SE emission. This should give rise to a bright contrast in the image, as has been observed for Si or CdSe. ${ }^{[11]}$ However, the dark contrast observed in the case 
of InGaN NWs suggests a different mechanism, where SEs in the conduction band lose energy during transit to the surface and they do not reach the detector because of an energy deficit, as explained by Cho et al. ${ }^{[12]}$ In this case, scattering processes with photo-generated electron-hole pairs are most likely responsible for the energy loss. As the effective cross-section for the scattering of SEs with conduction electrons is much higher than that with valence electrons, ${ }^{[12]} \mathrm{a}$ decrease in SE emission and, subsequently, a low contrast is observed.

We further compare our imaging results with carrier dynamics that we obtained from ultrafast pump-probe spectroscopic measurements. Figure 4a shows the transient absorption (TA) spectra of the nanowires in the ns- $\mu$ s region after $515 \mathrm{~nm}$ optical excitation. Clear ground state bleaching is observed at 460 and $603 \mathrm{~nm}$ due to the removal of the occupied valence band population, as confirmed from the peak position of the steady-state absorption spectra of the nanowires (Figure S2). The kinetics of the ground state bleach recovery at $460 \mathrm{~nm}$ (measured with ns-TA spectroscopy), which is fitted with a double exponential function, yields two time components: $\sim 2$ ns and $\sim 101$ ns (Figure 4B, inset). The short component matches the band-edge excitonic recombination time of InGaN that has been reported in literature. ${ }^{[55-56]}$ The long component probably arises from the recombination via a localized state, where the overlap between electron and hole wavefunctions is considerably low, or via some intra-bandgap trap sites, which may arise in indium rich regions. ${ }^{[57-58]}$ When the TA kinetics is compared with the kinetics obtained from the S-UEM, it is observed that within a time window of 4 ns (Figure 4b), both kinetics are exactly the same, which clearly proves the authenticity of the data obtained from S-UEM. 


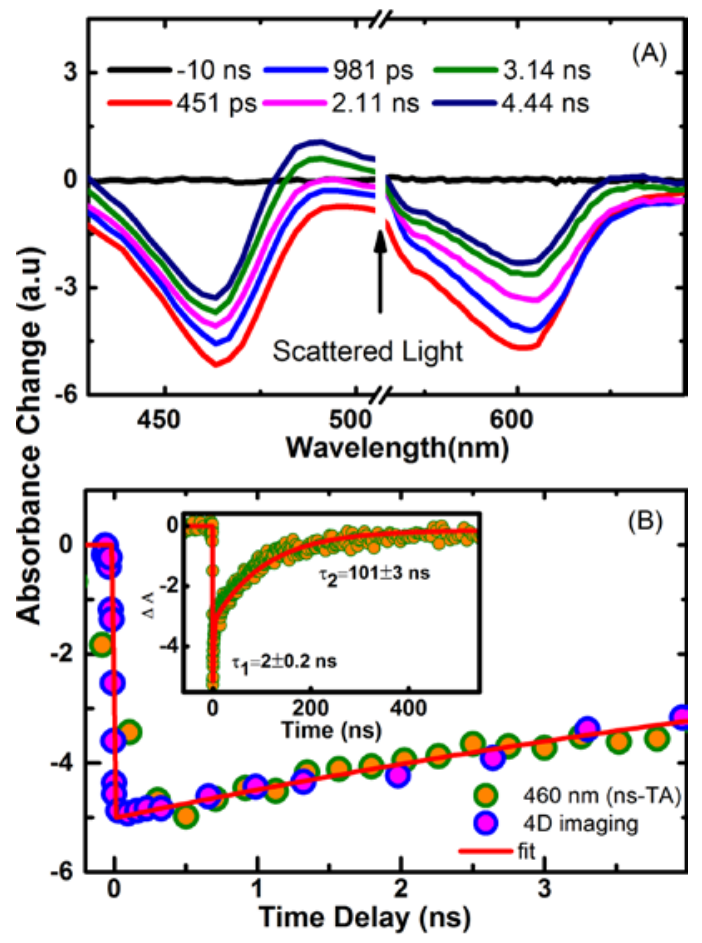

Figure 4. (A) The TA spectra for InGaN NWs in the ns region at the indicated time delays. (B) Kinetics that were obtained at $460 \mathrm{~nm}$ from the TA spectra in comparison with the kinetics that were obtained from the S-UEM. Both data sets overlap with each other exactly in the 4 ns timewindow. Inset shows the complete kinetics for the ground state bleach recovery with a longer time window, as obtained from the TA measurement. The solid lines are the best fit for the experimental data.

To get further insight into carrier relaxation and diffusion process, we performed numerical modelling for the SE signal variation in time and space. We assume that the sample material is homogeneous in lateral direction, i.e. possible inhomogeneity of physical properties is effectively averaged off. Hence the model involves effective diffusion coefficients for electrons and holes, which most likely is determined by charge transfer between individual nanowires as this process is expected to be much slower than the carrier migration inside the nanowires. As the SE signal is proportional to local free electron concentration on the surface of the materials, we take into 


\section{WILEY-VCH}

account two main processes taking place after laser pulse excitation of the nanowires. The first process is the diffusion of the photo-excited free electrons and holes across the boundaries of the nanowire array resulting in visible size increase of the area generating SE signal. The second process is the recombination of free carriers resulting in the overall SE signal decrease. To simplify the task we assume that the excited area has a circular shape and the carrier diffusion is isotropic such that we can neglect the shape variation. This also allows reducing the diffusion problem to the one-dimensional one. Then the space and time variation of the free electron and hole concentrations $n_{e}$ and $n_{h}$ can be described by the following equations

$$
\begin{aligned}
& \frac{d n_{e}(x, t)}{d t}=D_{e} \frac{d^{2} n_{e}(x, t)}{d x^{2}}-\gamma_{1} n_{e}(x, t)-\gamma_{2} n_{e}(x, t) \cdot n_{h}(x, t) \\
& \frac{d n_{h}(x, t)}{d t}=D_{h} \frac{d^{2} n_{h}(x, t)}{d x^{2}}-\gamma_{1} n_{h}(x, t)-\gamma_{2} n_{e}(x, t) \cdot n_{h}(x, t)
\end{aligned}
$$

where, all concentrations are normalized on the initial concentration at its maximum $N_{\mathrm{e} 0}, D_{e}$ and $D_{h}$ are diffusion coefficients of electrons and holes, respectively, $\gamma_{1}$ and $\gamma_{2}$ are monomolecular and bimolecular recombination rates, respectively. Both these rates have dimensionality s ${ }^{-1}$. To translate $\gamma_{2}$ to the common dimensionality $\mathrm{cm}^{3} \mathrm{~s}^{-1}$, it has to be divided by $N_{e 0}$. The latter can be estimated using the excitation pulse energy, the pulse cross section and the material absorption coefficient. 

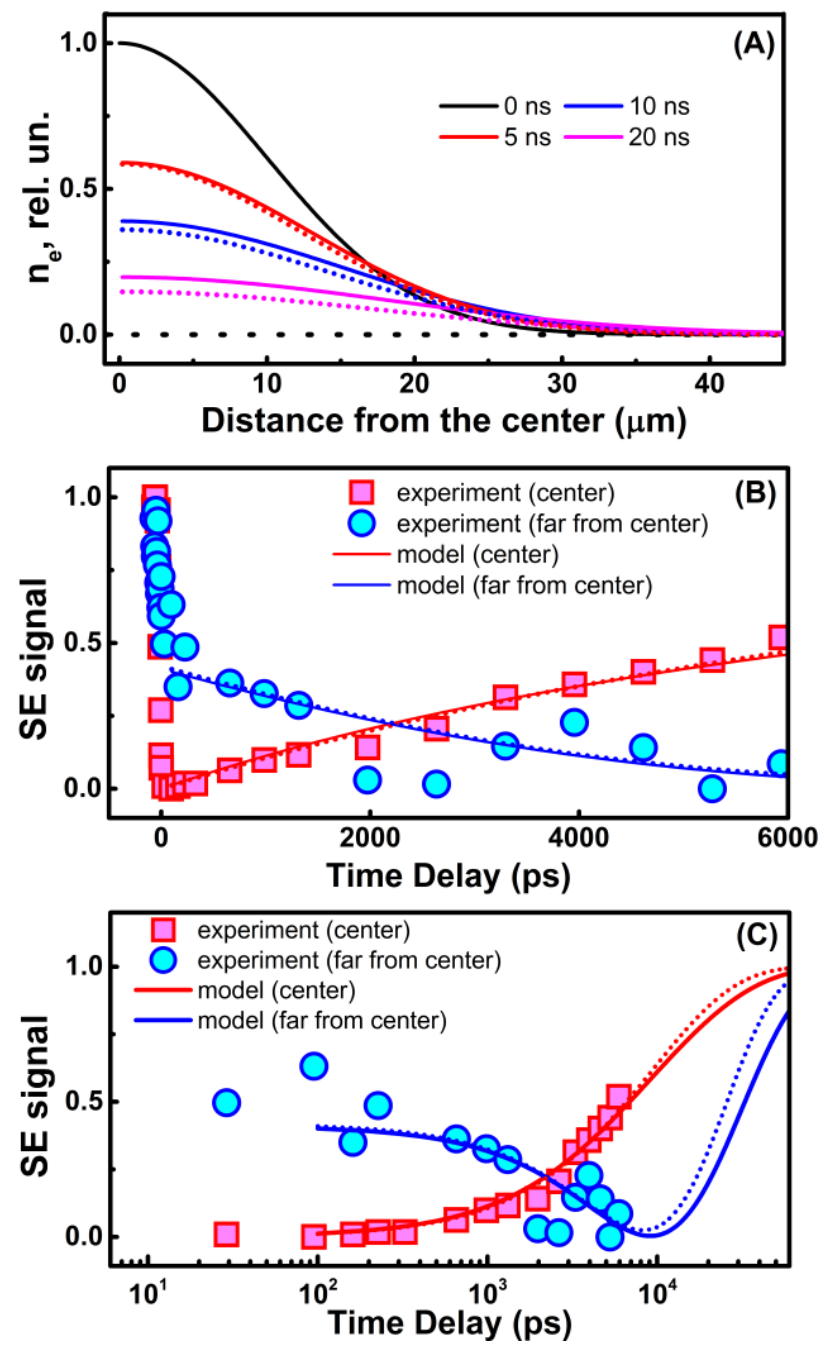

Figure 5. (A) Distribution of electronic concentration at different time moments. (B) Numerical fit for the kinetics of SE intensity evolution at the center and outside of laser footprint obtained by simulation. (C) Numerical fit for the kinetics of SE intensity evolution at extended time scale from the center and outside of laser footprint obtained by simulation. The model parameters used for simulation are $D_{e}=D_{h}=45 \mathrm{~cm}^{2} \mathrm{~s}^{-1}, \gamma_{1}=4 \cdot 10^{7} \mathrm{~s}^{-1}\left(\gamma_{1}=7 \cdot 10^{7} \mathrm{~s}^{-1}\right.$-for dotted lines $), \gamma_{2}=4 \cdot 10^{7}$ $s^{-1}\left(\gamma_{2}=0-\right.$ for dotted lines).

Eq (1) has been solved numerically with the initial data $n_{e}(x, 0)=n_{h}(x, 0)=\exp \left(-x^{2} / 2 \sigma^{2}\right)$ with $\sigma=10^{-3} \mathrm{~cm}$ for various values of the parameters $D_{e}, D_{h}, \gamma_{1}$ and $\gamma_{2}$ to fit the experimental data for 
normalized SE signal in the center and at the edge of the excited area. The normalized simulated signal at the center $(\mathrm{x}=0)$ has been taken as $1-n_{e}(0, t)$, while that at the edge (at the distance $\mathrm{x}=2.5 \cdot \sigma$ from the center) was proportional to $1-\alpha . n_{e}(2.5 \sigma, t)$ with $\alpha$ being the fit parameter. We found that for the parameter values $D_{e}=45 \mathrm{~cm}^{2} \mathrm{~s}^{-1}, \gamma_{1}=7 \cdot 10^{7} \mathrm{~s}^{-1}$ and $\gamma_{2} \approx 0$, the best fit (shown by solid lines in Figure 5B) for both SE signals (at $x=0$ and $x=2.5 \cdot \sigma$ ) can be obtained, suggesting that holes are not taking part in the SE signals. It is worth pointing out that nearly the similar quality fit (see solid lines in Figure 5B) can be obtained for the values $\gamma_{1}=\gamma_{2}=4 \cdot 10^{7} \mathrm{~s}^{-1}$ and $D_{e}=$ $D_{h}=45 \mathrm{~cm}^{2} \mathrm{~s}^{-1}$ suggesting that the simulation results are not very sensitive to the presence of bimolecular recombination and the presence of holes per se, which again implies that the modelling results wouldn't be sensitive also to the triple-molecular electronic relaxation, i.e., the Auger processes. Therefore, we can conclude that the leading mechanism of non-radiative energy loss involves mono-molecular electronic relaxation. The higher value of electronic diffusion coefficient $D_{e}$ compared to that used above $\left(26 \mathrm{~cm}^{2} \mathrm{~s}^{-1}\right)$ is due to the presence of recombination. The overall decrease in electronic density due to recombination requires much higher value of $D_{e}$ to account for the same increase in the SE signal at the periphery region as in the case without the recombination. This is illustrated in Figure 5A by the decreasing and broadening distribution of electronic density. The presence of recombination is really necessary for reproducing the experimental shape of the SE signal in the center (red lines in Figure 5B). To summarize, the model provides a good description of the SE signals in both experimentally measured spatial regions, i.e. in the center and at the periphery of the laser spot (see Figure 5B). This description allows rather accurate estimation of the electron diffusion coefficient while the description quality is not very sensitive to all other parameters, as their cumulative effect is mutually compensated (compare solid and dotted lines in Figure 5 B and C). 


\section{WILEY-VCH}

In conclusion, we report on the first real-space imaging of the charge carrier dynamics on the surface of InGaN NWs using 4D S-UEM. This technique provides the unique opportunity to directly visualize surface dynamics selectively, including carrier recombination and diffusion in real space and time, thus providing access to a territory that is beyond the reach of either static imaging or time-resolved laser spectroscopy/microscopy. Moreover, we identified the possible deactivation channels and the mechanism of secondary electron energy loss in the system. Numerical modelling for the SE signal variation in time and space further gave an accurate estimation of the carrier relaxation and diffusion process, which were consistent with the experimental results. Real-time visualization of the charge carrier dynamics on the surface not only provides a basic understanding of semiconductor physics but also enables intricate mapping of energy loss pathways, which are a major hurdle for advancing the performance of InGaN in optoelectronic devices.

\section{ASSOCIATED CONTENT}

Supporting Information. Ray diagram of 4D SUEM optical setup, Cross-sectional SEM image, steady-state UV-Vis absorbance and Time-resolved SE images at 30 and $1 \mathrm{keV}$ electrons for CdSe single crystal and its thin film.

\section{AUTHOR INFORMATION}

Corresponding Author

* Email: omar.abdelsaboor@kaust.edu.sa* 
Notes

The authors declare no competing financial interests.

\section{Supporting Information}

Supporting Information is available from the Wiley Online Library or from the author.

\section{Acknowledgements}

§These authors contributed equally to this work. The work was supported by the King Abdullah University of Science and Technology (KAUST) and King Abdulaziz City for Science and Technology TIC (Technology Innovation Center) for Solid-State Lighting at KAUST. TKN and BSO gratefully acknowledge contribution from Pallab Bhattacharya, University of Michigan, USA. T.K.N. and D.P. gratefully acknowledge Rami T. Elafandy (Photonics Laboratory, KAUST) for the effort and assistance in scanning electron microscopy experiments.

Received: ((will be filled in by the editorial staff))

Revised: ((will be filled in by the editorial staff)) Published online: ((will be filled in by the editorial staff))

\section{REFERENCES}

[1] D. J. Flannigan, A. H. Zewail, Acc. Chem. Res. 2012, 45, 1828.

[2] A. H. Zewail, Science 2010, 328, 187.

[3] V. A. Lobastov, R. Srinivasan, A. H. Zewail, Proc. Natl. Acad. Sci. 2005, 102, 7069.

[4] H. Petek, ACS Nano 2014, 8, 5.

[5] H. S. Park, J. S. Baskin, O.-H. Kwon, A. H. Zewail, Nano Lett. 2007, 7, 2545.

[6] D.-S. Yang, O. F. Mohammed, A. H. Zewail, Proc. Natl. Acad. Sci. 2010, 107, 14993.

[7] M. S. Grinolds, V. A. Lobastov, J. Weissenrieder, A. H. Zewail, Proc. Natl. Acad. Sci. 2006, 103, 18427.

[8] A. Yurtsever, A. H. Zewail, Science 2009, 326, 708.

[9] F. Carbone, O.-H. Kwon, A. H. Zewail, Science 2009, 325, 181.

[10] O.-H. Kwon, A. H. Zewail, Science 2010, 328, 1668.

[11] O. F. Mohammed, D.-S. Yang, S. K. Pal, A. H. Zewail, J. Am. Chem. Soc. 2011, 133, 7708. 


\section{WILEY-VCH}

[12] J. Cho, T. Y. Hwang, A. H. Zewail, Proc. Natl. Acad. Sci. 2014, 111, 2094.

[13] J. Sun, V. A. Melnikov, J. I. Khan, O. F. Mohammed, J. Phys. Chem. Lett. 2015, 6, 3884.

[14] E. Najafi, T. D. Scarborough, J. Tang, A. Zewail, Science 2015, 347, 164.

[15] E. M. Grumstrup, M. M. Gabriel, E. M. Cating, C. W. Pinion, J. D. Christesen, J. R. Kirschbrown, E. L. Vallorz, J. F. Cahoon, J. M. Papanikolas, J. Phys. Chem. C 2014, 118, 8634.

[16] M. M. Gabriel, J. R. Kirschbrown, J. D. Christesen, C. W. Pinion, D. F. Zigler, E. M. Grumstrup, B. P. Mehl, E. E. M. Cating, J. F. Cahoon, J. M. Papanikolas, Nano Lett. 2013, 13, 1336.

[17] A. Salant, M. Shalom, Z. Tachan, S. Buhbut, A. Zaban, U. Banin, Nano Lett. 2012, 12, 2095.

[18] M. T. McDowell, S. W. Lee, I. Ryu, H. Wu, W. D. Nix, J. W. Choi, Y. Cui, Nano Lett. 2011, 11, 4018.

[19] C. K. Chan, R. N. Patel, M. J. O’Connell, B. A. Korgel, Y. Cui, ACS Nano 2010, 4, 1443.

[20] Y. M. Zuev, J. S. Lee, C. Galloy, H. Park, P. Kim, Nano Lett. 2010, 10, 3037.

[21] L. Venkataraman, Y. S. Hong, P. Kim, Phys. Rev. Lett. 2006, 96, 076601.

[22] T. A. Su, H. Li, V. Zhang, M. Neupane, A. Batra, R. S. Klausen, B. Kumar, M. L. Steigerwald, L. Venkataraman, C. Nuckolls, J. Am. Chem. Soc. 2015, 137, 12400.

[23] Y.-J. Doh, K. N. Maher, L. Ouyang, C. L. Yu, H. Park, J. Park, Nano Lett. 2008, 8, 4552.

[24] D. Yu, J. Wu, Q. Gu, H. Park, J. Am. Chem. Soc. 2006, 128, 8148.

[25] H. Eshet, R. Baer, D. Neuhauser, E. Rabani, J. Phys. Chem. Lett. 2014, 5, 2580.

[26] H. Ding, N. Pan, C. Ma, Y. Wu, J. Li, H. Cai, K. Zhang, G. Zhang, W. Ren, J. Li, Y. Luo, X. Wang, J. G. Hou, Adv. Mater. 2014, 26, 3035.

[27] W. Zhou, X. Dai, T.-M. Fu, C. Xie, J. Liu, C. M. Lieber, Nano Lett. 2014, 14, 1614.

[28] Z. Jiang, Q. Qing, P. Xie, R. Gao, C. M. Lieber, Nano Lett. 2012, 12, 1711.

[29] K. T. Delaney, P. Rinke, C. G. Van de Walle, Appl. Phys. Lett. 2009, 94, 191109.

[30] X. Ni, X. Li, J. Lee, S. Liu, V. Avrutin, Ü. Özgür, H. Morkoç, A. Matulionis, J. Appl. Phys. 2010, 108, 033112.

[31] K.-G. Gan, C.-K. Sun, S. P. DenBaars, J. E. Bowers, Appl. Phys. Lett. 2004, 84, 4675.

[32] M. Müller, A. Paarmann, R. Ernstorfer, Nat Commun 2014, 5, 5292.

[33] M. Bernardi, D. Vigil-Fowler, C. S. Ong, J. B. Neaton, S. G. Louie, Proc. Natl. Acad. Sci. 2015, 112, 5291.

[34] C. Hahn, Z. Zhang, A. Fu, C. H. Wu, Y. J. Hwang, D. J. Gargas, P. Yang, ACS Nano 2011, 5, 3970.

[35] W. Guo, M. Zhang, A. Banerjee, P. Bhattacharya, Nano Lett. 2010, 10, 3355.

[36] F. Qian, Y. Li, S. Gradecak, H.-G. Park, Y. Dong, Y. Ding, Z. L. Wang, C. M. Lieber, Nat. Mater. 2008, 7, 701.

[37] Y. Dong, B. Tian, T. J. Kempa, C. M. Lieber, Nano Lett. 2009, 9, 2183.

[38] T. Kuykendall, P. Ulrich, S. Aloni, P. Yang, Nat. Mater. 2007, 6, 951.

[39] A. David, M. J. Grundmann, Appl. Phys. Lett. 2010, 96, 103504.

[40] S. Hammersley, D. Watson-Parris, P. Dawson, M. J. Godfrey, T. J. Badcock, M. J. Kappers, C. McAleese, R. A. Oliver, C. J. Humphreys, J. Appl. Phys. 2012, 111, 083512.

[41] C. H. Wang, S. P. Chang, W. T. Chang, J. C. Li, Y. S. Lu, Z. Y. Li, H. C. Yang, H. C. Kuo, T. C. Lu, S. C. Wang, Appl. Phys Lett. 2010, 97, 181101.

[42] F. Bertazzi, M. Goano, E. Bellotti, Appl. Phys. Lett. 2010, 97, 231118. 
[43] E. Kioupakis, P. Rinke, K. T. Delaney, C. G. Van de Walle, Appl. Phys. Lett. 2011, 98, 161107.

[44] E. Bellotti, F. Bertazzi, M. Goano, J. Appl. Phys. 2007, 101, 123706.

[45] J. Iveland, L. Martinelli, J. Peretti, J. S. Speck, C. Weisbuch, Phys. Rev. Lett. 2013, 110, 177406.

[46] C. X. Wang, K. Tsubaki, N. Kobayashi, T. Makimoto, N. Maeda, Appl. Phys. Lett. 2004, 84, 2313.

[47] L. Hsu, R. E. Jones, S. X. Li, K. M. Yu, W. Walukiewicz, J Appl. Phys. 2007, 102, 073705.

[48] Z. Guo, J. S. Manser, Y. Wan, P. V. Kamat, L. Huang, Nat Commun 2015, 6, 7471.

[49] C. Zhao, T. K. Ng, A. Prabaswara, M. Conroy, S. Jahangir, T. Frost, J. O'Connell, J. D. Holmes, P. J. Parbrook, P. Bhattacharya, B. S. Ooi, Nanoscale 2015, 7, 16658.

[50] S. Nakamura, Science 1998, 281, 956.

[51] M. Takashi, Y. Motokazu, N. Shuji, Jpn. J. Appl. Phys. 1999, 38, 3976.

[52] S. Nakamura, Semicond. Sci. Techol. 1999, 14, R27.

[53] P. Kivisaari, J. Oksanen, J. Tulkki, Appl. Phys. Lett. 2013, 103, 031103.

[54] L. Reimer, Scanning Electron Microscopy, Vol. 45, 2 ed., Springer.

[55] S. Nakamura, M. Senoh, S. i. Nagahama, N. Iwasa, T. Yamada, T. Matsushita, Y. Sugimoto, H. Kiyoku, Appl. Phys. Lett. 1996, 69, 1568.

[56] H. P. T. Nguyen, M. Djavid, S. Y. Woo, X. Liu, A. T. Connie, S. Sadaf, Q. Wang, G. A. Botton, I. Shih, Z. Mi, Sci. Rep. 2015, 5, 7744.

[57] T. Li, A. M. Fischer, Q. Y. Wei, F. A. Ponce, T. Detchprohm, C. Wetzel, Appl. Phys. Lett. 2010, 96, 031906.

[58] E. S. Jeon, V. Kozlov, Y. K. Song, A. Vertikov, M. Kuball, A. V. Nurmikko, H. Liu, C. Chen, R. S. Kern, C. P. Kuo, M. G. Craford, Appl. Phys. Lett. 1996, 69, 4194. 


\section{Table of content}

This paper describes a breakthrough in the development of four-dimensional (4D) scanning ultrafast electron microscopy for real- time and space imaging of secondary electron energy loss and carrier diffusion the surface of nanowires array as a model system, providing access to a territory that is beyond the reach of either static electron imaging or any time-resolved laser spectroscopy.

Real-Space Visualization of Energy Loss and Carrier Diffusion in Semiconductor Nanowire Array using Four-Dimensional Electron Microscopy

\section{TOC graphic}

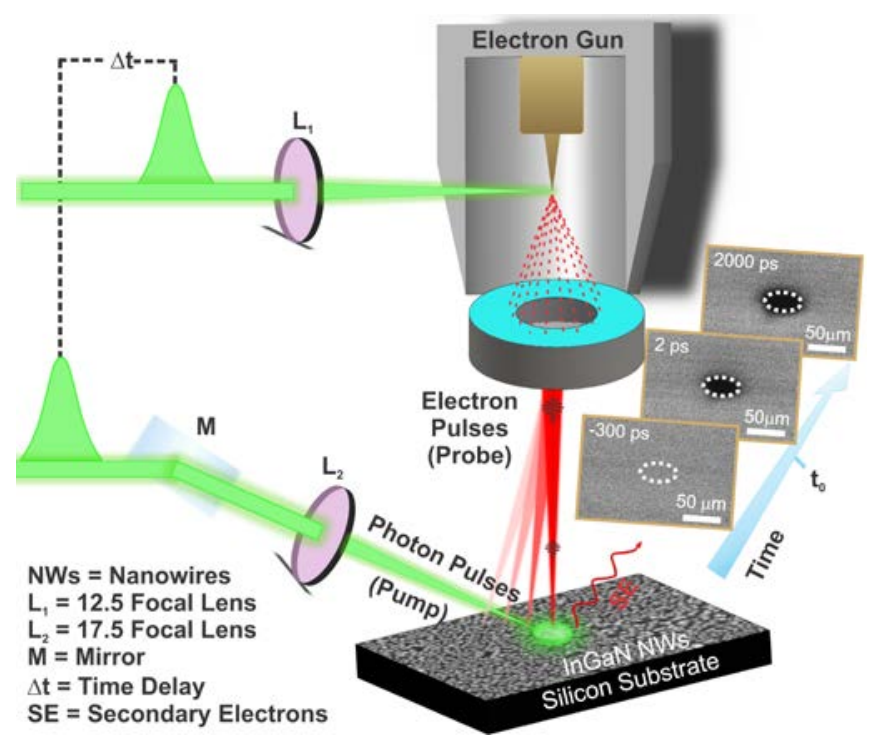




\section{Supporting Information}

\section{Real-Space Visualization of Energy Loss and Carrier Diffusion in Semiconductor Nanowire Array using Four-Dimensional Electron Microscopy}

Riya Bose, ${ }^{\S, 1}$ Jingya Sun, ${ }^{\S, 1}$ Jafar I. Khan, ${ }^{1}$ Basamat S. Shaheen, ${ }^{1}$ Aniruddha Adhikari, ${ }^{1}$ Tien Khee Ng, ${ }^{2}$ Victor M. Burlakov, ${ }^{3}$ Manas R. Parida, ${ }^{1}$ Davide Priante, ${ }^{2}$ Alain Goriely, ${ }^{3}$ Boon S. Ooi, ${ }^{2}$ Osman M. Bakr, ${ }^{1}$ Omar F. Mohammed*1

Dr. R. Bose, Dr. J. Sun, Dr. J. I. Khan, Dr. A. Adhikari, B. S. Shaheen, Prof. O. M. Bakr, Prof. O. F. Mohammed, Solar and Photovoltaics Engineering Research Center, Division of Physical Sciences and Engineering, King Abdullah University of Science and Technology, Thuwal 23955-6900, Kingdom of Saudi Arabia, D. Priante, Dr. T. K. Ng, Prof. B. S. Ooi, Photonics Laboratory, Computer, Electrical, and Mathematical Sciences and Engineering KAUST, Thuwal, 23955-6900, Kingdom of Saudi Arabia

Dr. V. M. Burlakov and Prof. A. Goriely, Mathematical Institute, University of Oxford, Woodstock Road, Oxford OX2 6GG, UK, 


\section{Experimental Section}

\section{Fabrication of InGaN nanowires}

The InGaN nanowires crystals were grown using Veeco Gen-II plasma assisted molecular beam epitaxy (PAMBE) on silicon substrates using a process similar to our previous report. ${ }^{[1]}$ Prior to nanowires crystal growth, the silicon substrates were cleaned in hydrofluoric acid solution to remove surface oxide. The sample is then heated in two stages, in the adjoining load-lock and transfer vacuum chambers, for removing the adsorbed molecules on the substrate. The pristine substrate is then transferred into the growth chamber for further oxide desorption at an elevated temperature of $800^{\circ} \mathrm{C}$. The InGaN nanowires are then grown catalyst free at temperatures less than $600^{\circ} \mathrm{C}$, with the indium and gallium vapor flux pre-calibrated to obtain the photoluminescence peak emission wavelength above $600 \mathrm{~nm}$. The background pressure of the growth chamber was kept at $\sim 1 \times 10^{-5}$ Torr, which is sustained by supplying the nitrogen plasma species required for the formation of InGaN nanowires crystals. After crystal growth, the sample is cooled down and removed from the ultra-high vacuum chambers for subsequent experimentation.

\section{Control experiment to confirm the origin of dark contrast at negative time delays of SUEM experiment}

To further confirm the origin of the dark contrast in the negative time, we did two different control S-UEM experiments, (1) we imaged the thin film of CdSe (about $60 \mathrm{~nm}$ thickness) and bulk single crystal (about $5 \mathrm{~mm}$ thickness) of CdSe; (2) we changed the energy of the electron beam from $30 \mathrm{keV}$ to $1 \mathrm{keV}$. It is well known that that when the energy of the electron beam is 1 $\mathrm{keV}$, the penetration depth is much smaller. In $1 \mathrm{keV}$ experiment, we do not observe the dark signal at the negative time in contrast to $30 \mathrm{keV}$, which clearly shows dark signal at negative time (Figure S3). Similarly CdSe films with $\sim 60 \mathrm{~nm}$ thickness does not show dark signal at negative time contrary to the single crystal of CdSe (Figure S4). Both these results suggest that 


\section{WILEY-VCH}

the negative signal must have originated from plasmon excited carriers that are generated from deeper areas of the sample. It is worthy to mention that CdSe was used for the control experiments since it is much easier to control the thickness for CdSe film compared to InGaN NWs.

\section{Instrumentation}

Steady-state spectroscopy: Absorption spectra were measured on a Varian Cary 6000i UV-VisNIR spectrophotometer with integrating sphere.

Time-Resolved Spectroscopy: $\mu$ s-TA spectroscopy was carried out using a pump-probe EOS spectrometer. The fundamental output came from a Ti:sapphire femtosecond regenerative amplifier operating at $800 \mathrm{~nm}$ with $35 \mathrm{fs}$ pulses and a repetition rate of $1 \mathrm{kHz}$. Spectrally tunable (240-2600 nm) femtosecond pulses generated by an Optical Parametric Amplifier (Light Conversion LTD) was used as the pump (excitation). A two-channel probe (probe-reference) method is used where the probe beam is split into two before passing through the sample- one travels through sample and the other is sent directly to the reference spectrometer that monitors the fluctuations in the probe beam intensity. ${ }^{2}$ 


\section{Supporting Figures}

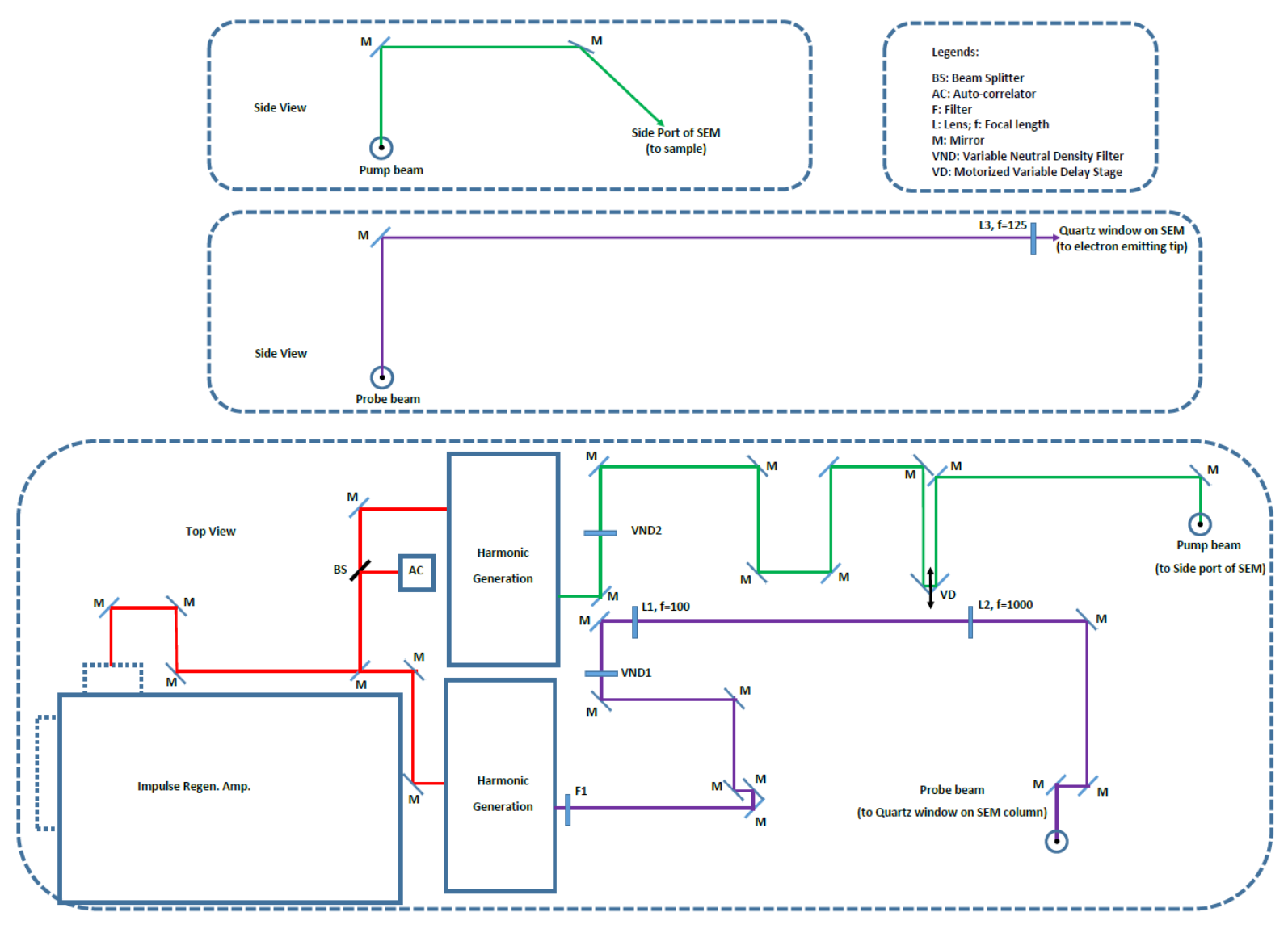

Figure S1. Ray diagram of 4D SUEM optical setup.
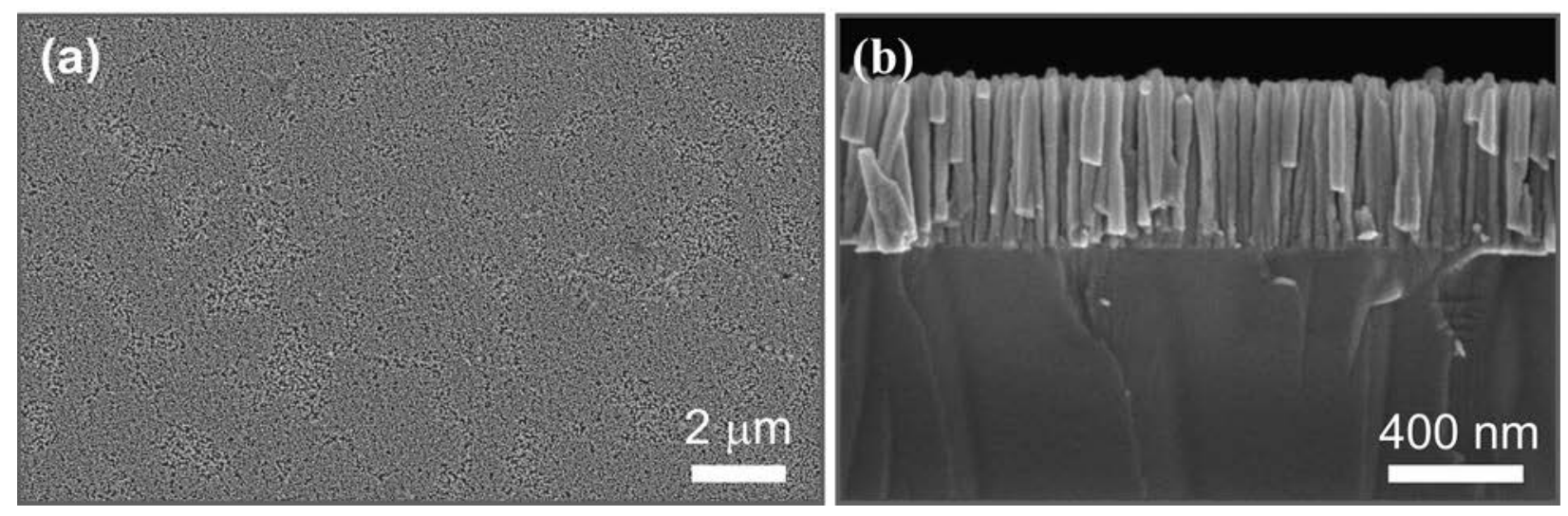

Figure S2. (a) Top view SEM image of InGaN nanowires grown on silicon substrate with a density of $\sim 4 \times 10^{10} \mathrm{~cm}^{-2}$, (b) Cross section SEM image of InGaN nanowires. 


\section{WILEY-VCH}

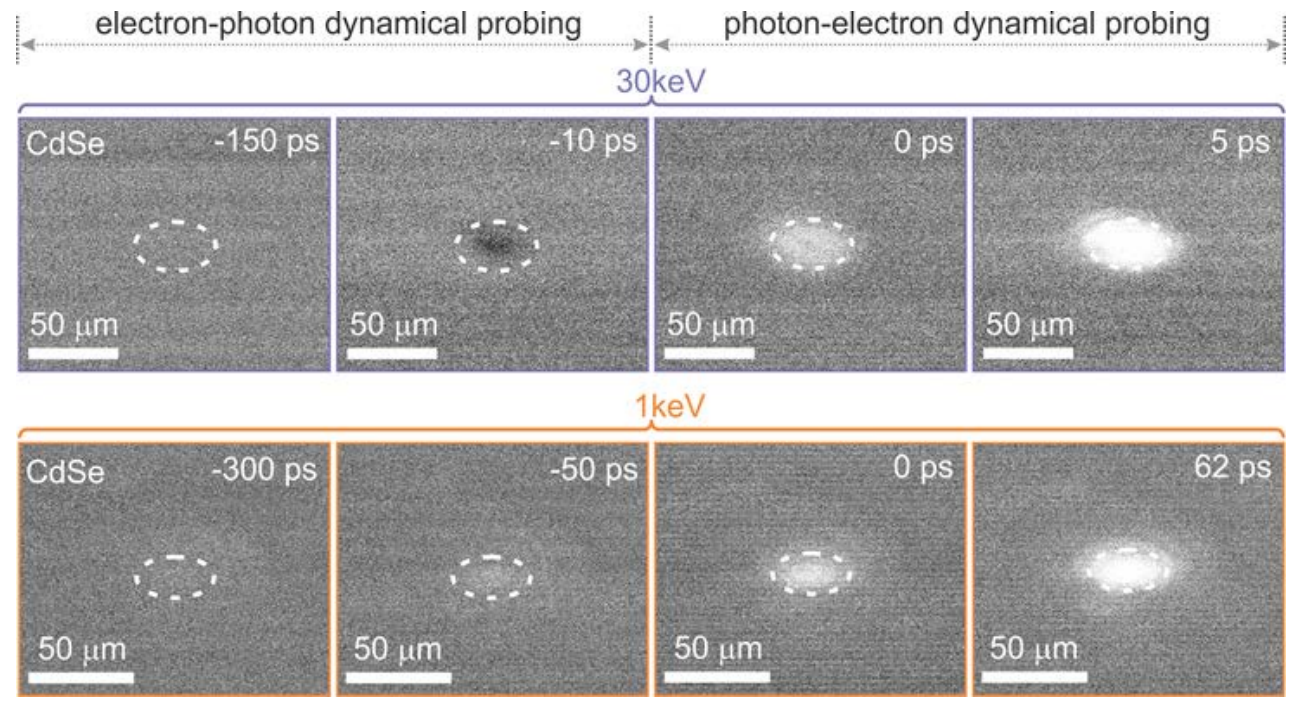

Figure S3. Time-resolved SE images of CdSe at the indicated time delays at energy of the electron beam being $30 \mathrm{keV}$ (top panel) and $1 \mathrm{keV}$ (bottom panel). The dashed ellipses indicate the footprint of the clocking optical beam on the specimen $(\sim 40 \mu \mathrm{m})$.

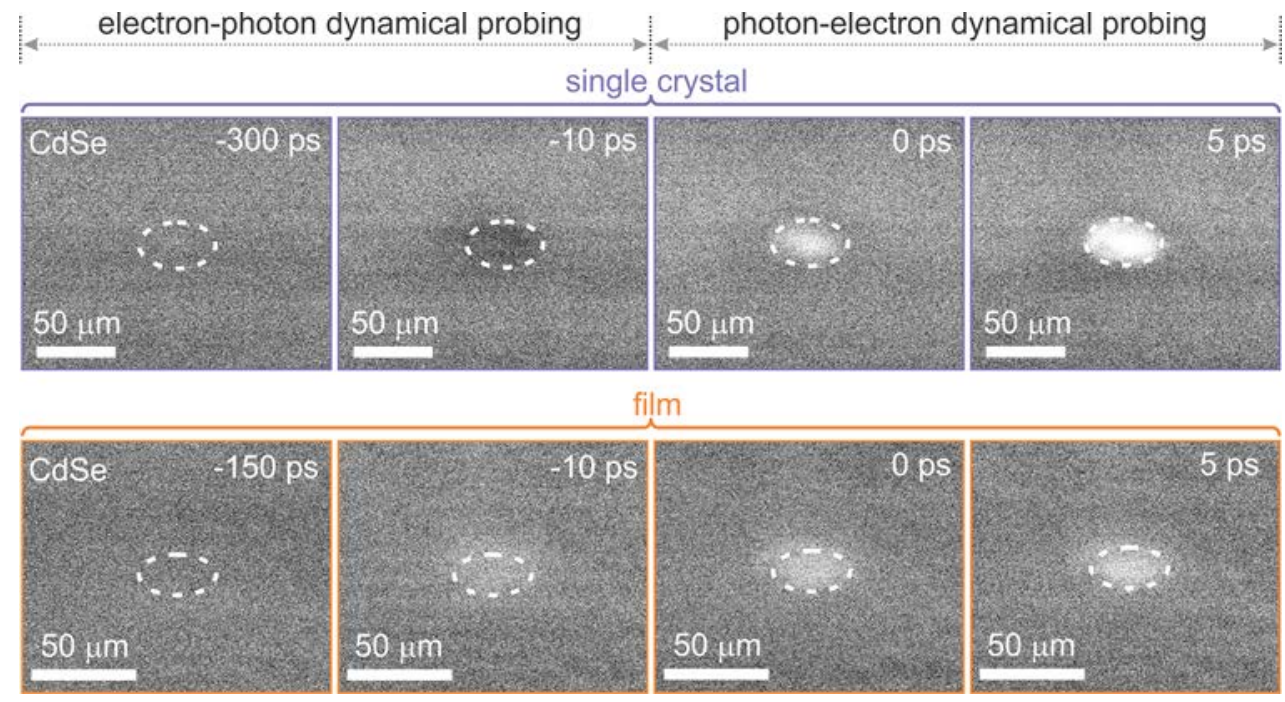

Figure S4. Time-resolved SE images of CdSe single crystal (upper panel) and film (bottom panel) with $60 \mathrm{~nm}$ thickness at the indicated time delays. The dashed ellipses indicate the footprint of the clocking optical beam on the specimen $(\sim 40 \mu \mathrm{m})$. 

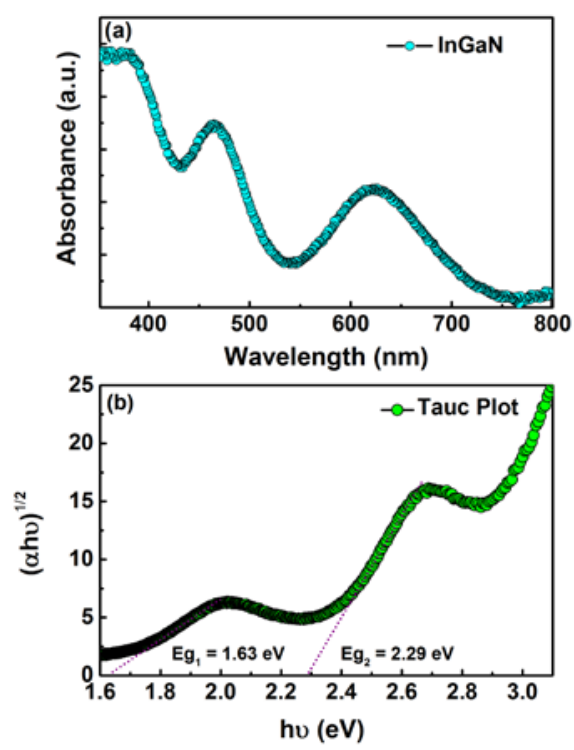

Figure S5. (a) Steady state UV-Vis absorbance spectra of InGaN nanowires (b) Tauc plot to determine the bandgap of the nanowires.

\section{References:}

[1] T. Frost, S. Jahangir, E. Stark, S. Deshpande, A. Hazari, C. Zhao, B. S. Ooi and P. Bhattacharya, Nano Lett. 2014, 14, 4535.

[2] R. Bose, G. H. Ahmed, E. Alarousu, M. R. Parida, A. L. Abdelhady, O. M. Bakr, O. F. Mohammed, J. Phys. Chem. C, 2015, 119, 3439. 